\title{
Conference Paper \\ Experimental Analysis of Microstructured Steel Surfaces for Wet Tribological Applications in the Low Velocity Regime
}

\author{
Mateusz Chlipala, ${ }^{1}$ Johannes Schneider, ${ }^{2}$ and Volker Schulze ${ }^{1}$ \\ ${ }^{1}$ wbk Institute of Production Science, Karlsruhe Institute of Technology (KIT), Kaiserstrasse 12, 76131 Karlsruhe, Germany \\ ${ }^{2}$ Institute for Applied Materials (IAM), Karlsruhe Institute of Technology (KIT), Kaiserstrasse 12, 76131 Karlsruhe, Germany \\ Correspondence should be addressed to Mateusz Chlipala; mateusz.chlipala@kit.edu
}

Received 1 August 2014; Accepted 27 November 2014

Academic Editor: Alfons Fischer

This Conference Paper is based on a presentation given by Mateusz Chlipala at "European Symposium on Friction, Wear, and Wear Protection" held from 6 May 2014 to 8 May 2014 in Karlsruhe, Germany.

Copyright (C) 2015 Mateusz Chlipala et al. This is an open access article distributed under the Creative Commons Attribution License, which permits unrestricted use, distribution, and reproduction in any medium, provided the original work is properly cited.

\begin{abstract}
The surface topography plays an important role in the design of a function-optimised surface. Therefore, the influence of topography with microsized structures produced by laser surface texturing (LST) is experimentally examined under lubricated sliding conditions. The structured specimens were made of AISI 51200 (DIN 100Cr6) hardened to about 800 HV. Concerning the requirements of tribological testing without any debris caused by the preprocessing, the structuring was carried out using a picosecond laser system (Trumpf TruMicro) with 6 ps pulse duration. A laboratory pin-on-disc tribometer (Plint TE-92 HS) was used for the tests, which were run under wet conditions with counterbodies made of bronze and steel at a nominal contact pressure of up to $4 \mathrm{MPa}$ and sliding speeds between 0.04 and $2.0 \mathrm{~m} / \mathrm{s}$. Furthermore, start-stop cycles with accelerating and decelerating shares were used to simulate an automotive start-stop system. In the tribological experiments, a significant reduction of the friction coefficient was observed compared to sliding pairs without microstructured pin surfaces. Whereas no measureable wear occurred on the steel pins and discs, the bronze discs showed a significant amount of wear and the microstructures on the pin surfaces mated against bronze discs were almost completely filled with wear debris.
\end{abstract}

\section{Introduction}

Since the transport sector accounts for a big share in anthropogenic $\mathrm{CO}_{2}$ emissions, there is a need to reduce fuel consumption in this area. In passenger cars, about one-third of the fuel energy is used to overcome friction. These friction losses can be broken down to braking and rolling resistance on one side and losses in the engine and transmission on the other side [1]. There are possibilities to reduce friction by engineered surfaces particularly in the area of engine and transmission.

Besides the early work of Zum Gahr [2] who showed the importance of topography on the frictional system recent research has shown that a structured topography can lead to positive effects regarding a tribological system. Microdimples or generally structures separated from each other on surfaces in lubricated systems can produce a wedge flow effect which leads to an increased hydrodynamic pressure in the contact which is able to reduce friction significantly $[3,4]$. Conversely interacting channel-like structures on the surface can increase friction coefficient caused by an increased oil flow out of the contact area [5]. Also in dry experiments structured surfaces can lead to beneficial conditions. In such case surface dimples can be filled with particles resulting from wear during the running-in period of the system [6]. Furthermore in slow velocity regimes with high load surface dimples can act as lubricant reservoirs and therefore reduce the risk of wear in mixed lubrication regimes [7]. Therefore, in this paper, a variation of size values of the structures 
was carried out in order to examine their influence on the tribological system.

\section{Surface Engineering}

2.1. Surface Texturing. The structured specimens were made of AISI 51200 (DIN 100Cr6) hardened to about $800 \mathrm{HV}$ and ground to a roughness of about $0.1 \mu \mathrm{m}\left(R_{a}\right)$. In order to receive structures which meet the requirements for tribological testing, the surface texturing was carried out with a picosecond laser (Trumpf TruMicro) with 6 ps pulse duration and a wavelength of $1030 \mathrm{~nm}$. Because of the short pulse duration it is possible to machine the structures with no burrs resulting from melted material as they would occur using laser systems with pulse durations in the nanoseconds regime [8]. The influence of laser parameters was reviewed in order to analyze the resulting geometrical structure sizes and possible burr formation on the structure sides. The experiments were carried out with a repetition rate of $20 \mathrm{kHz}$ in line mode. Line mode in this case means that dimple structures are ablated while moving the beam over the surface fast enough to receive a distance between the structures (negative pulse overlap). Because of the fast movement and the long time span between two pulses at one specific position it is possible to avoid heat accumulation effects or particle shielding. The structures were afterwards analyzed using a confocal microscope (Nanofocus) with lateral accuracy of $0.3 \mu \mathrm{m}$ and axial accuracy of $10 \mathrm{~nm}$.

2.2. Results of Laser Surface Texturing. As already shown theoretically in [8] or experimentally, for example, in [9], the ablation rate $L$ for low peak laser fluences $\left(\phi_{0}<1 \mathrm{~J} / \mathrm{cm}^{2}\right)$ per pulse can be described with $1 / \alpha$ as the optical penetration depth of the electromagnetic wave inside the material and the ablation threshold $\phi_{\mathrm{th}}$ by the expression

$$
L=\alpha^{-1} \ln \left(\frac{\phi_{0}}{\phi^{\alpha}{ }_{\text {th }}}\right)
$$

while for higher peak fluences $\left(\phi_{0}>1 \mathrm{~J} / \mathrm{cm}^{2}\right)$ the logarithmic dependence can be described by

$$
L=\gamma \ln \left(\frac{\phi_{0}}{\phi^{\gamma}{ }_{\text {th }}}\right),
$$

where $\gamma$ stands for the heat penetration depth inside the material. While working with high peak fluences even at ultrashort pulse durations, a so-called strong ablation takes place where molten material appears [10]. Adding the number of repetitions $n$ to (1) results in the ablated depth $h$ of a dimple structure in the gentle regime,

$$
h=n \alpha^{-1} \ln \left(\frac{\phi_{0}}{\phi_{\text {th }}}\right),
$$

which can be used to model the ablated depth based on the laser parameters.

The influence of different pulse energies (and resulting peak fluence) and number of repetitions on the depth of

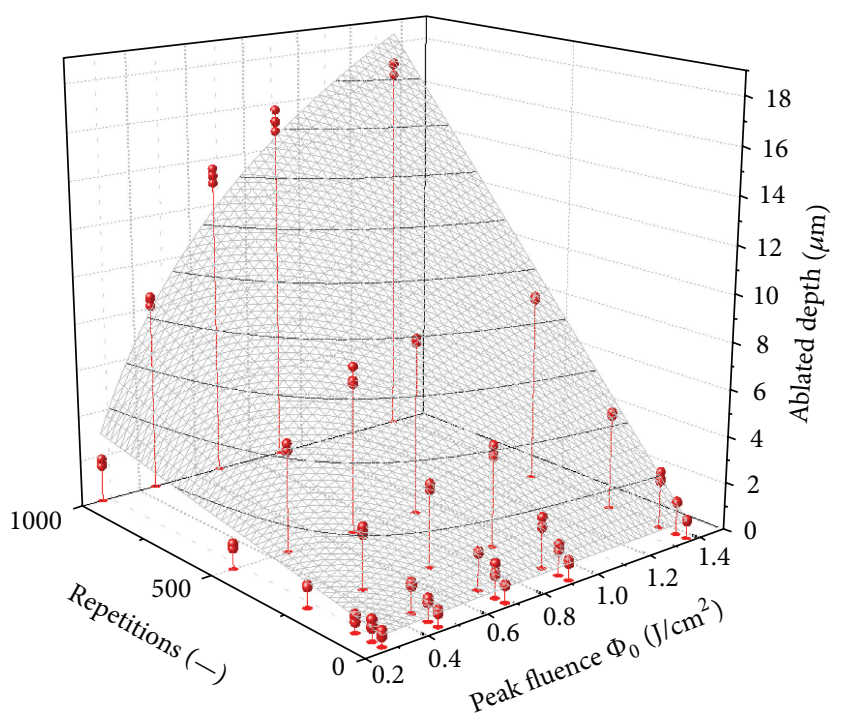

FIgUre 1: The ablated depth of dimples in AISI 52100 as a function of the peak fluence and the number of repetitions per position.

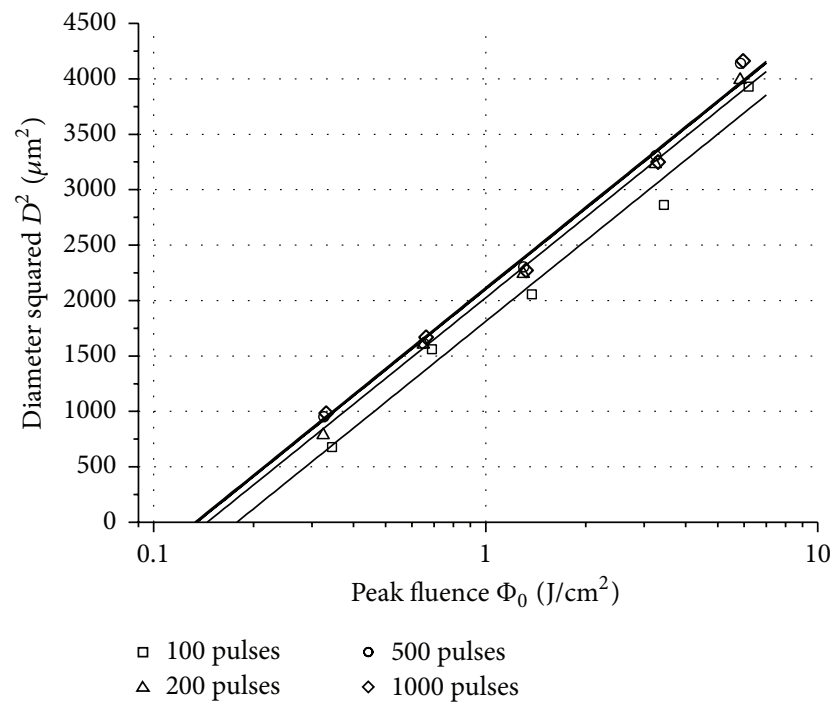

FIGURE 2: Squared diameter of the ablated dimples as a function of the peak laser fluence, LIPSS, at the edge of a dimple structure.

the dimples is shown in Figure 1. The experimental results follow here quite reasonable $\left(R^{2}=0.98\right)$ the theoretical values calculated by using (3) with the fitted values $\alpha^{-1} \approx 9.5 \mathrm{~nm}$ and $\phi_{\text {th }}=0.21 \mathrm{~J} / \mathrm{cm}^{2}$.

The influence of peak laser fluence and the repetitions on the ablated squared diameter area on the surface are shown in Figure 2. The values were obtained by converting the measured results of the square of the dimple diameter versus the logarithmic pulse energy. Because of the linear dependence of the pulse energy and the peak laser fluence the focus diameter can be easily calculated [11].

As already noted in [9] for up to 100 pulses the damage threshold (intersection between extrapolated squared diameter and $x$-axis) in steel decreases with increasing repetitions 


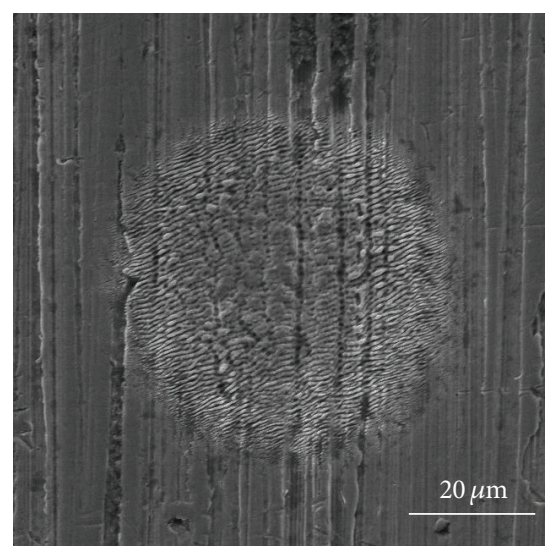

(a)

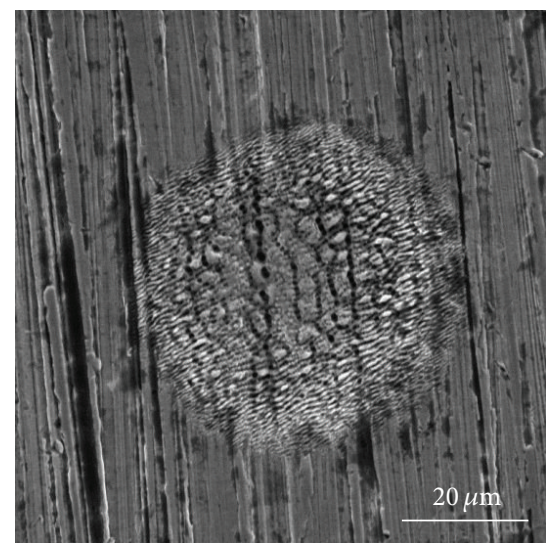

(b)

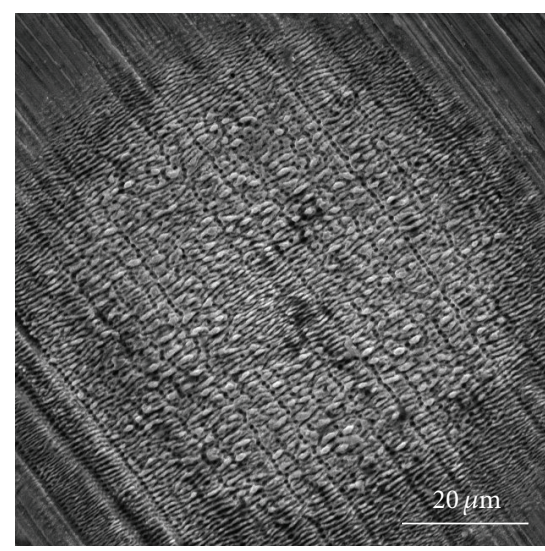

(c)

FIGURE 3: SEM pictures of dimples showing rough surfaces due to "Laser Induced Periodic Surface Structures" (LIPSS). (a) Structure 1. (b) Structure 2. (c) Structure 3.

caused by incubation effects. Incubation appears to be the result of the storage cycle of thermal stress-strain energy induced by a laser pulse [12]. This effect can be described by the following equation [9]

$$
\phi_{\mathrm{th}}(n)=\phi_{\mathrm{th}}(1) n^{(S-1)}
$$

with an additional incubation factor $S$ leading to a decrease of the threshold from the very first laser pulse on the surface $\phi_{\mathrm{th}}(1)$. Equation (4) would lead to a nonrealistic small threshold value for a very high number of repetitions but fits fairly well the examined parameter range of up to 1000 repetitions $(S=0.88)$ in this and other work, for example, [9].

Like shown in Figure 2 for more than 200 pulse repetitions the damage threshold converges into the resulting thresholds with 500 or 1000 pulses. This means that the maximum achievable dimple diameter is reached after 200 repetitions. Like in other work [13] the generation of LIPSS (Laser Induced Periodic Structures) was observed near the ablation threshold. Because of the Gaussian intensity distribution this roughening of the surface takes place on the sides of the ablated dimple structure (Figure 3 ) leading to a presumption of increased absorption in this area, thus reducing the ablation threshold. This could be an explanation of the observed incubation effect besides other theories based on the stored stress-strain energy resulting from the heated material [12]. With that information at hand a specific dimple structure size (depth, diameter) can be predicted and set.

The variable dimple parameters in this work were the structured area, the dimple diameter, and depth. These structures were ablated with similar framework conditions like the preliminary tests. The exemplary structures for the tribological tests shown in Figure 3 had a diameter of $54 \mu \mathrm{m}$ (structures 1 and 2 with a structured area ratio 20\%) and $100 \mu \mathrm{m}$ (structure 3 with a structured area ratio 35\%). The structured depth was $1.5 \mu \mathrm{m}$ ("1"), $2.8 \mu \mathrm{m}$ ("2"), and $1.1 \mu \mathrm{m}$ (“3”), respectively.
2.3. Tribological Tests. The structured steel pins were tribologically evaluated in unidirectional sliding contact using a laboratory pin-on-disc tribometer (Plint TE-92 HS) with counterbodies made of bearing bronze ( $\left.155 \mathrm{HV}, R_{a}=0.5 \mu \mathrm{m}\right)$ and steel AISI $51200\left(800 \mathrm{HV}, R_{a}=0.1 \mu \mathrm{m}\right)$. The tests were run under wet conditions (Shell Rimula R6 ME 5W$30 @ 80^{\circ} \mathrm{C}$ with a flow rate $720 \mathrm{~mL} / \mathrm{h}$ ) with normal loads of 60 and $120 \mathrm{~N}$ (contact pressure of 2 and $4 \mathrm{MPa}$ for a pellet surface diameter of $6 \mathrm{~mm}$ ). All tests were started with a normal load of $60 \mathrm{~N}$ at the highest speed of $2.0 \mathrm{~m} / \mathrm{s}$, which was then reduced down to $0.04 \mathrm{~m} / \mathrm{s}$ in 12 steps holding each for $120 \mathrm{~s}$. This Stribeck velocity ramp was repeated twice and afterwards 50 start-stop cycles with accelerating and decelerating shares (accelerating from 0 to $2 \mathrm{~m} / \mathrm{s}$ within $10 \mathrm{~s} / /$ running at $2 \mathrm{~m} / \mathrm{s}$ for $5 \mathrm{~s} / /$ decelerating from 2 to $0 \mathrm{~m} / \mathrm{s}$ within $10 \mathrm{~s}$ ) were run to simulate an automotive start-stop system. The complete test procedure consisting of 2 Stribeck velocity ramps and 50 start-stop cycles was then repeated with a normal load of $120 \mathrm{~N}$.

Figure 4 shows Stribeck curves derived from tests with ground and microstructured pins against both C93200 (ASTM B505) bearing bronze and steel AISI 51200 discs at constant sliding speeds. The lowest friction coefficient for steel/steel as well as steel/bronze sliding pairs was found for couples with microtextured pins. In contact with bronze discs at $60 \mathrm{~N}$ the tribological behaviour of pins with surface structures 1 and 2 resulted in a reduced friction coefficient compared to the ground reference pin. The use of pins with structure 3 however resulted in higher friction below $1 \mathrm{~m} / \mathrm{s}$ (Figure 4(a)). For the steel/steel sliding pairs structures 1 and 3 led to a decreased friction coefficient and structure 2 led to an increased friction coefficient (Figure 4(b)). The normal load of $120 \mathrm{~N}$ resulted in higher friction coefficients of all tested sliding pairs and only the pin surfaces with the "best" structures, structure 2 for bronze and structure 1 for steel, resulted in a significantly reduced friction coefficient in relation to the ground reference surface (Figures 4(c) 


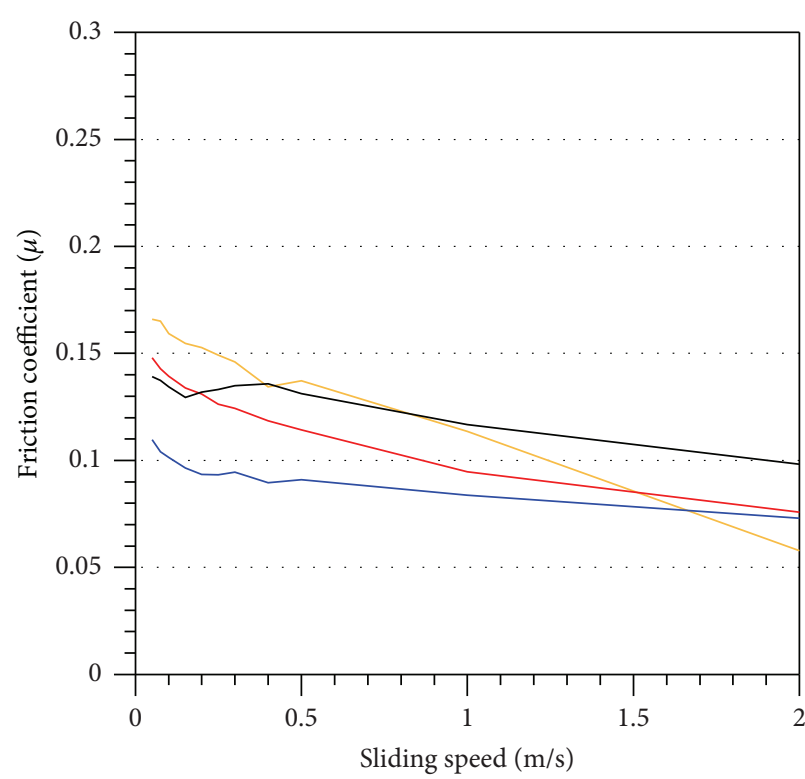

(a) $60 \mathrm{~N}$

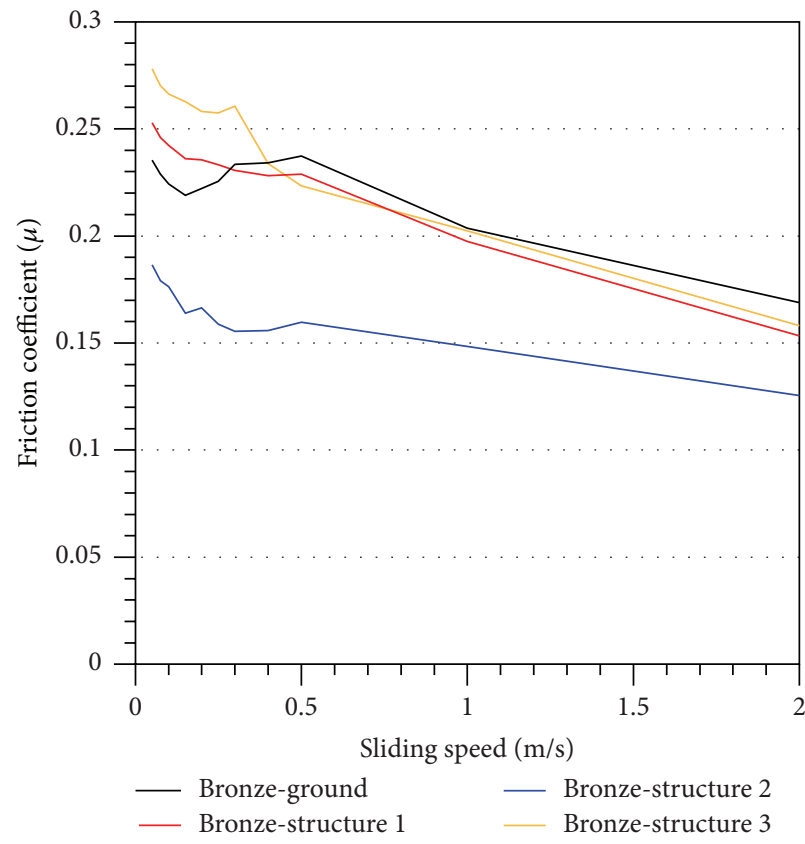

(c) $120 \mathrm{~N}$

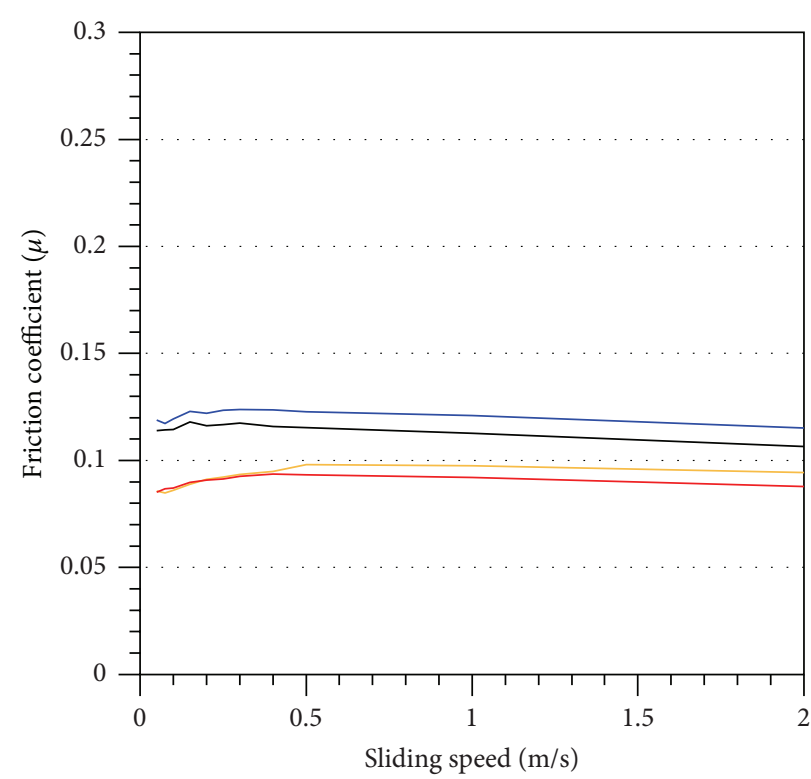

(b) $60 \mathrm{~N}$

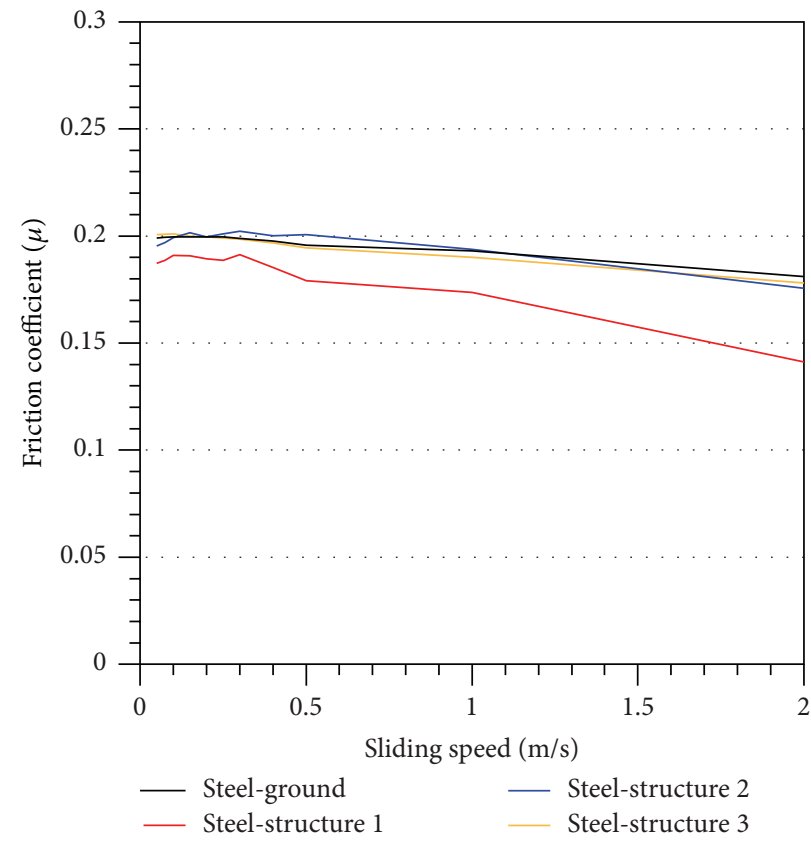

(d) $120 \mathrm{~N}$

FIGURE 4: Friction coefficient versus sliding speed for pins with 3 different structure sizes and a ground reference pin mated against (a, c) bronze and (b, d) steel during Stribeck velocity ramps at normal loads of (a, b) $60 \mathrm{~N}$ and (c, d) $120 \mathrm{~N}$.

and $4(\mathrm{~d}))$. In the case of bronze discs the average friction reduction achieved by the "best" structure 2 was between 25 and $30 \%$ at both normal loads. Only for sliding speeds of $0.1 \mathrm{~m} / \mathrm{s}$ and below the positive effect was less pronounced. The effectiveness of the structured topography was lower for the steel/steel sliding pairs. Here the "best" structure 1 led to a friction reduction of 20 to $25 \%$ at $60 \mathrm{~N}$ and of 5 to $10 \%$ at $120 \mathrm{~N}$ for sliding speeds up to $1 \mathrm{~m} / \mathrm{s}$.

The positive effect of a microstructured surface was also evident during the start-stop tests. Figure 5 exemplary shows acceleration curves from start-stop tests at $60 \mathrm{~N}$ for the sliding pairs with ground pins and pins with the "best" structures, respectively. The friction coefficients measured under the transient conditions were significantly higher than that under the steady state conditions shown before (see Figures 4 and 5), which could be attributed to oil film breakdown in the idle state and the need to build up the lubricant film during acceleration. For the steel sliding pair friction reduction with structure 1 amounted to about $15 \%$ over the complete speed range from 0.04 to $2 \mathrm{~m} / \mathrm{s}$ (Figure $5(\mathrm{~b})$ ). On the other side 


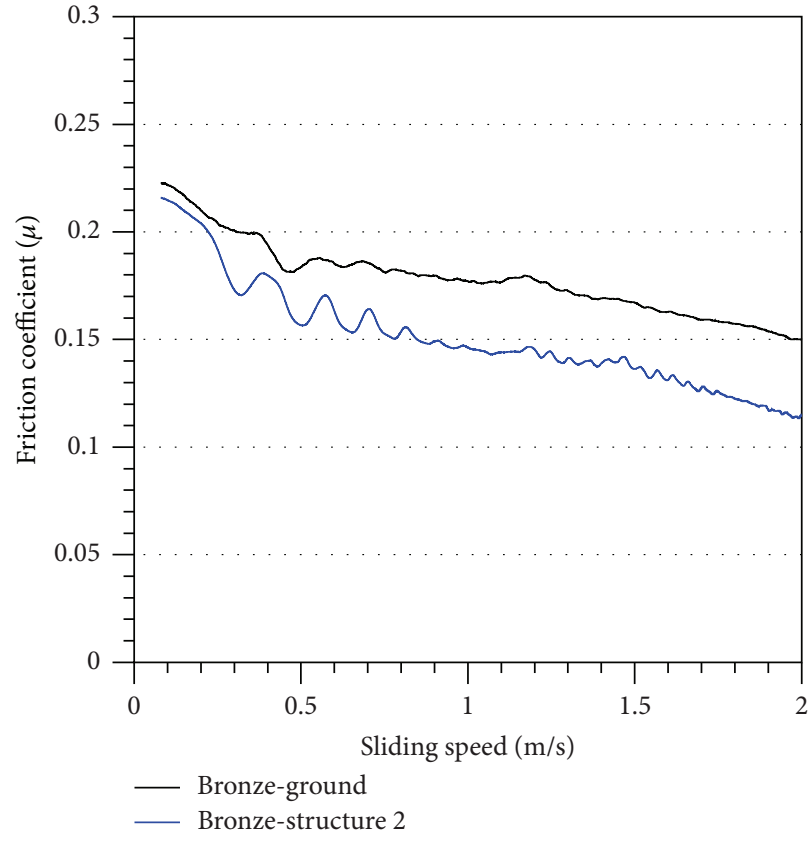

(a) $60 \mathrm{~N}$, acceleration

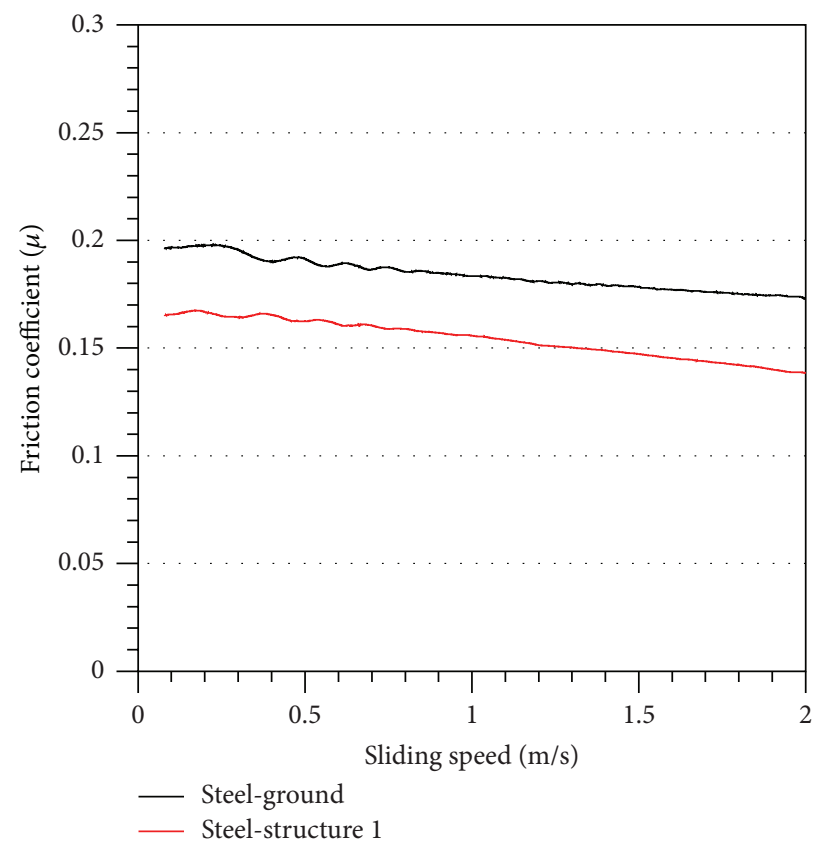

(b) $60 \mathrm{~N}$, acceleration

FIGURE 5: Friction coefficient versus sliding speed for ground and structured pins mated against (a) bronze and (b) steel during the acceleration ramp of the start-stop cycles at a normal load of $60 \mathrm{~N}$.

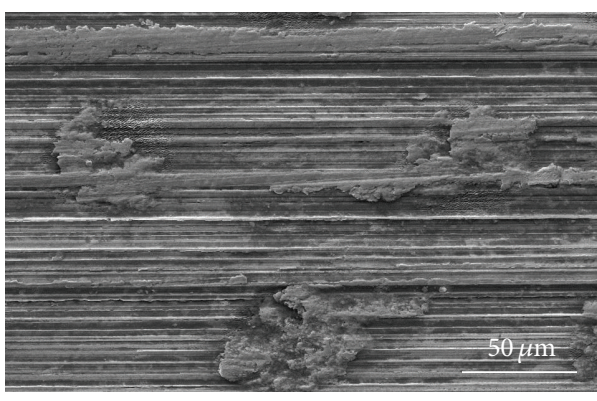

(a)

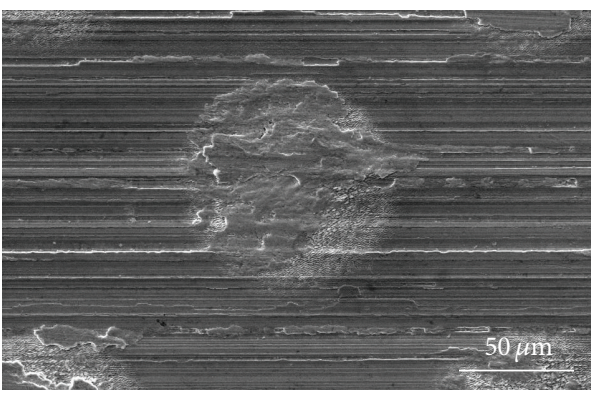

(c)

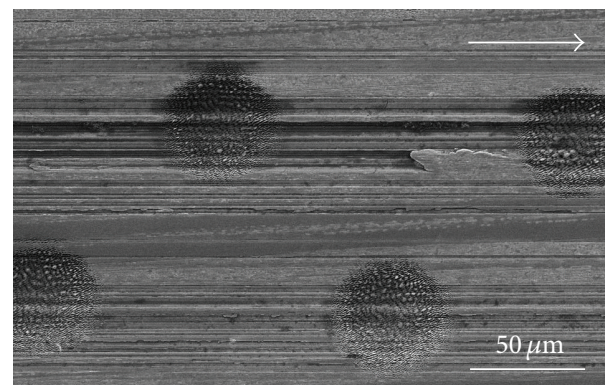

(b)

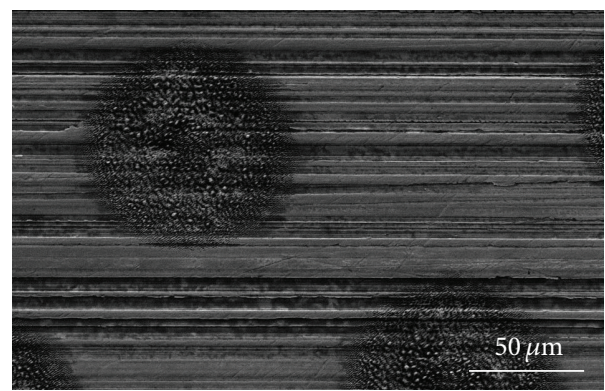

(d)

FIGURE 6: SEM pictures of microstructured pins after tribological tests in contact with (a, c) bronze and (b, d) steel discs. (a, b) Structure 1 and (c, d) structure 3 (arrow indicates the sliding direction).

structure 2 led to a friction reduction of about $15 \%$ only at sliding speeds of $0.5 \mathrm{~m} / \mathrm{s}$ and above for the steel/bronze sliding pair. A significant improvement of the frictional behavior for this sliding pair could not be detected below $0.2 \mathrm{~m} / \mathrm{s}$ (Figure 5(a)).
After the complete test procedure with overall 4 velocity ramps and 100 start-stop cycles at normal loads of 60 and $120 \mathrm{~N}$ no measureable wear occurred for the steel pins as well as the steel discs. On the other hand the bronze discs showed a significant linear wear in the range from $5.4 \mu \mathrm{m}$ (tested 


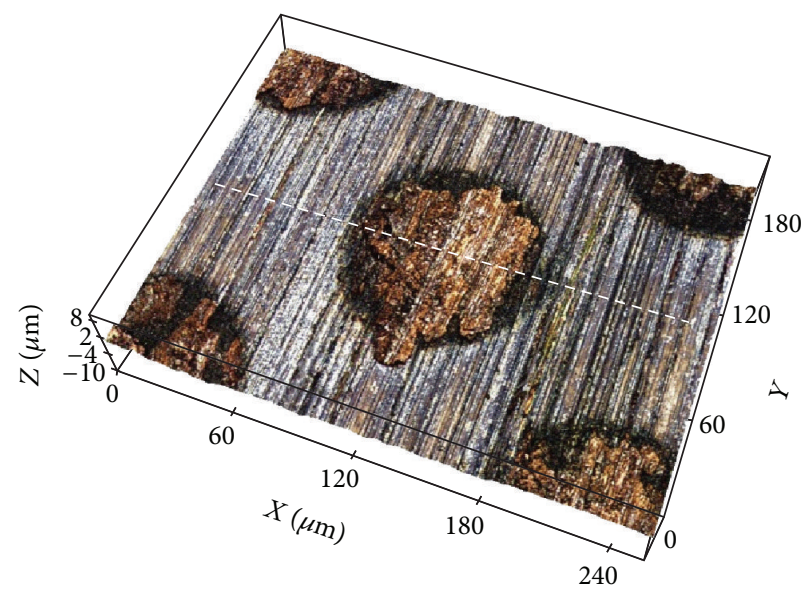

(a)

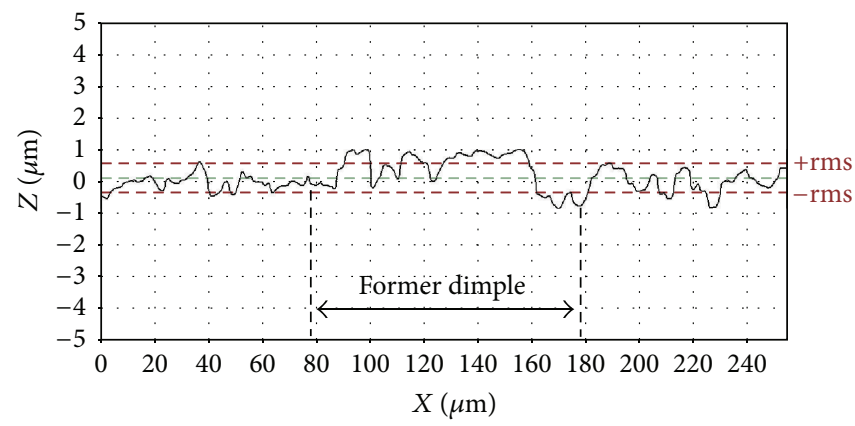

(b)

FIgURE 7: (a) Topography and (b) surface profile of pins with structure 3 after a tribological test in contact with bronze.

versus pin with structure 2) to $7.5 \mu \mathrm{m}$ (tested versus pin with structure 3 ).

The differences in wear behavior were also clearly visible on the surfaces of the pins after the tests. Figure 6 shows SEM pictures of worn steel pins after tests versus bronze (Figures 6(a) and 6(c)) and steel (Figures 6(b) and 6(d)) discs. The material worn away from the bronze discs was preferentially transferred to the dimples on the pin's surface, which were almost completely filled up with bronze. Actually the material's transfer led to the formation of veritable bumps on the pins (see Figure 7). The surfaces of the pins, which were run versus steel discs, were characterized by a slight smoothing of the surface roughness and the ground of the dimples was still clearly visible without notable wear particles (Figures 6(b) and 6(d)).

\section{Conclusion}

The results of the tribological tests clearly illustrate that, especially under mixed lubrication conditions, the optimized dimples can lead to a significant reduction of friction. The positive effect of the dimples can mainly be attributed to three mechanisms. In the low wear regime dimples can result in a positive hydrodynamic effect which results in an increased thickness of the oil film. The effectiveness of a specific dimple geometry thereby strongly depends on the experimental conditions (e.g., load, sliding speed, viscosity of the lubricant,...) $[5,14]$. The second positive effect is the trapping of wear particles. This effect is typically discussed to be of importance only during the running-in period until the dimples are either sealed by agglomerated wear particles or completely filled up with debris $[6,15]$. Our results showed that even completely filled dimples can have a positive hydrodynamic effect if a preferred adherence of wear particles at the dimples results in the formation of bumps. Such a reversed microtexturing was successfully used in hard disc drives $[16,17]$. The third effect often postulated in literature is the storing of lubricant in the dimples (secondary lubrication effect) $[7,18,19]$. The results of our start-stop experiments point out the importance of this effect, as empty dimples resulted in a significant friction reduction during the acceleration of the steel/steel sliding pair, whereas the completely filled dimples did not reduce friction at low sliding speeds for the steel/bronze sliding pairs. The negative effect of an increased local contact pressure at the bumps seems to overcompensate for the positive effect of the lubricant storage in the surrounding of the former dimples.

\section{Conflict of Interests}

The authors declare that there is no conflict of interests regarding the publication of this paper.

\section{References}

[1] K. Holmberg, P. Andersson, and A. Erdemir, "Global energy consumption due to friction in passenger cars," Tribology International, vol. 47, pp. 221-234, 2012.

[2] K.-H. Zum Gahr, Microstructure and Wear of Materials, Elsevier, Amsterdam, The Netherlands, 1987.

[3] I. Etsion, "Improving tribological performance of mechanical components by laser surface texturing," Tribology Letters, vol. 17, no. 4, pp. 733-737, 2004.

[4] A. Kovalchenko, O. Ajayi, A. Erdemir, G. Fenske, and I. Etsion, "The effect of laser surface texturing on transitions in lubrication regimes during unidirectional sliding contact," Tribology International, vol. 38, no. 3, pp. 219-225, 2005.

[5] R. Wahl, J. Schneider, and P. Gumbsch, "Influence of the real geometry of the protrusions in micro textured surfaces on frictional behaviour," Tribology Letters, vol. 47, no. 3, pp. 447453, 2012.

[6] A. Borghi, E. Gualtieri, D. Marchetto, L. Moretti, and S. Valeri, "Tribological effects of surface texturing on nitriding steel for high-performance engine applications," Wear, vol. 265, no. 7-8, pp. 1046-1051, 2008.

[7] I. Etsion, "State of the art in laser surface texturing," in Advanced Tribology, pp. 761-762, Springer, Berlin, Germany, 2010. 
[8] B. N. Chichkov, C. Momma, S. Nolte, F. Von Alvensleben, and A. Tünnermann, "Femtosecond, picosecond and nanosecond laser ablation of solids," Applied Physics A: Materials Science and Processing, vol. 63, no. 2, pp. 109-115, 1997.

[9] P. T. Mannion, J. Magee, E. Coyne, G. M. O'Connor, and T. J. Glynn, "The effect of damage accumulation behaviour on ablation thresholds and damage morphology in ultrafast laser micro-machining of common metals in air," Applied Surface Science, vol. 233, no. 1-4, pp. 275-287, 2004.

[10] S. Nolte, C. Momma, H. Jacobs et al., "Ablation of metals by ultrashort laser pulses," Journal of the Optical Society of America B: Optical Physics, vol. 14, no. 10, pp. 2716-2722, 1997.

[11] J. M. Liu, "Simple technique for measurements of pulsed Gaussian-beam spot sizes," Optics Letters, vol. 7, no. 5, pp. 196198, 1982.

[12] Y. Jee, M. F. Becker, and R. M. Walser, "Laser-induced damage on single-crystal metal surfaces," Journal of the Optical Society of America B, vol. 5, no. 3, pp. 648-659, 1988.

[13] J. Vincenc Obona, V. Ocelík, J. Z. P. Skolski et al., "On the surface topography of ultrashort laser pulse treated steel surfaces," Applied Surface Science, vol. 258, no. 4, pp. 1555-1560, 2011.

[14] D. Braun, C. Greiner, J. Schneider, and P. Gumbsch, "Efficiency of laser surface texturing in the reduction of friction under mixed lubrication," Tribology International, vol. 77, pp. 142-147, 2014.

[15] M. Varenberg, G. Halperin, and I. Etsion, "Different aspects of the role of wear debris in fretting wear," Wear, vol. 252, no. 11-12, pp. 902-910, 2002.

[16] J. Xu, H. Tokisue, H. Tanaka, and M. Matsumoto, "Contact vibration of micro-textured sliders," Journal of Tribology, vol. 124, no. 2, pp. 281-287, 2002.

[17] A. H. Tan and S. W. Cheng, "A novel textured design for hard disk tribology improvement," Tribology International, vol. 39, no. 6, pp. 506-511, 2006.

[18] X. Lu and M. M. Khonsari, "An experimental investigation of dimple effect on the stribeck curve of journal bearings," Tribology Letters, vol. 27, no. 2, pp. 169-176, 2007.

[19] S.-W. Lo and T.-C. Horng, "Lubricant permeation from micro oil pits under intimate contact condition," Journal of Tribology, vol. 121, no. 4, pp. 633-638, 1999. 

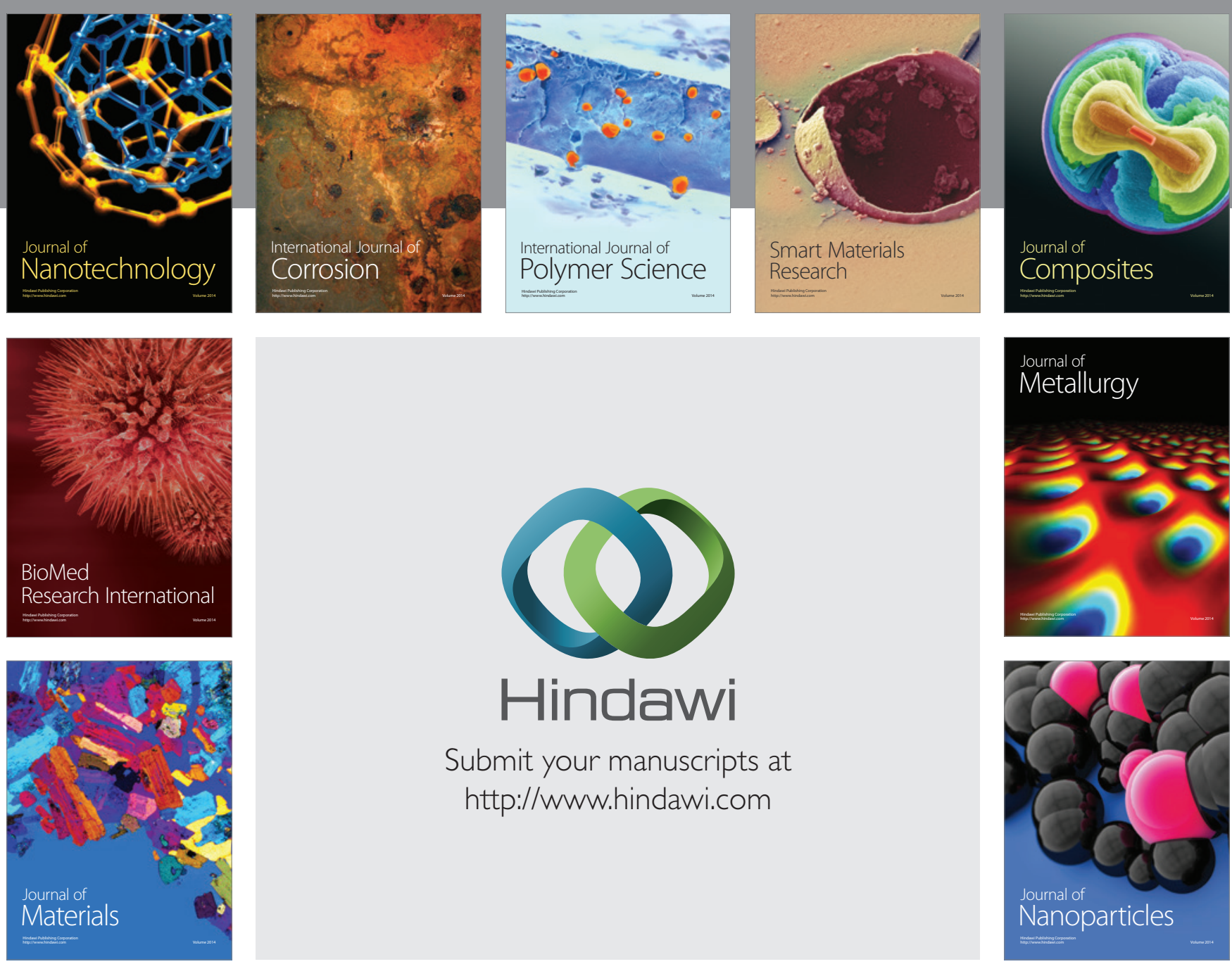

Submit your manuscripts at http://www.hindawi.com
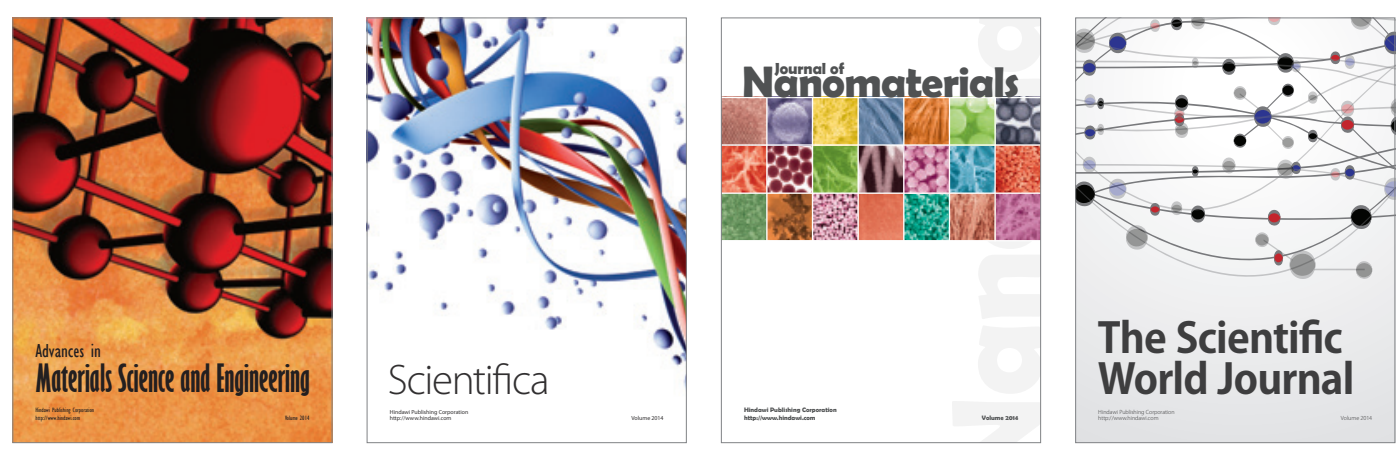

\section{The Scientific World Journal}
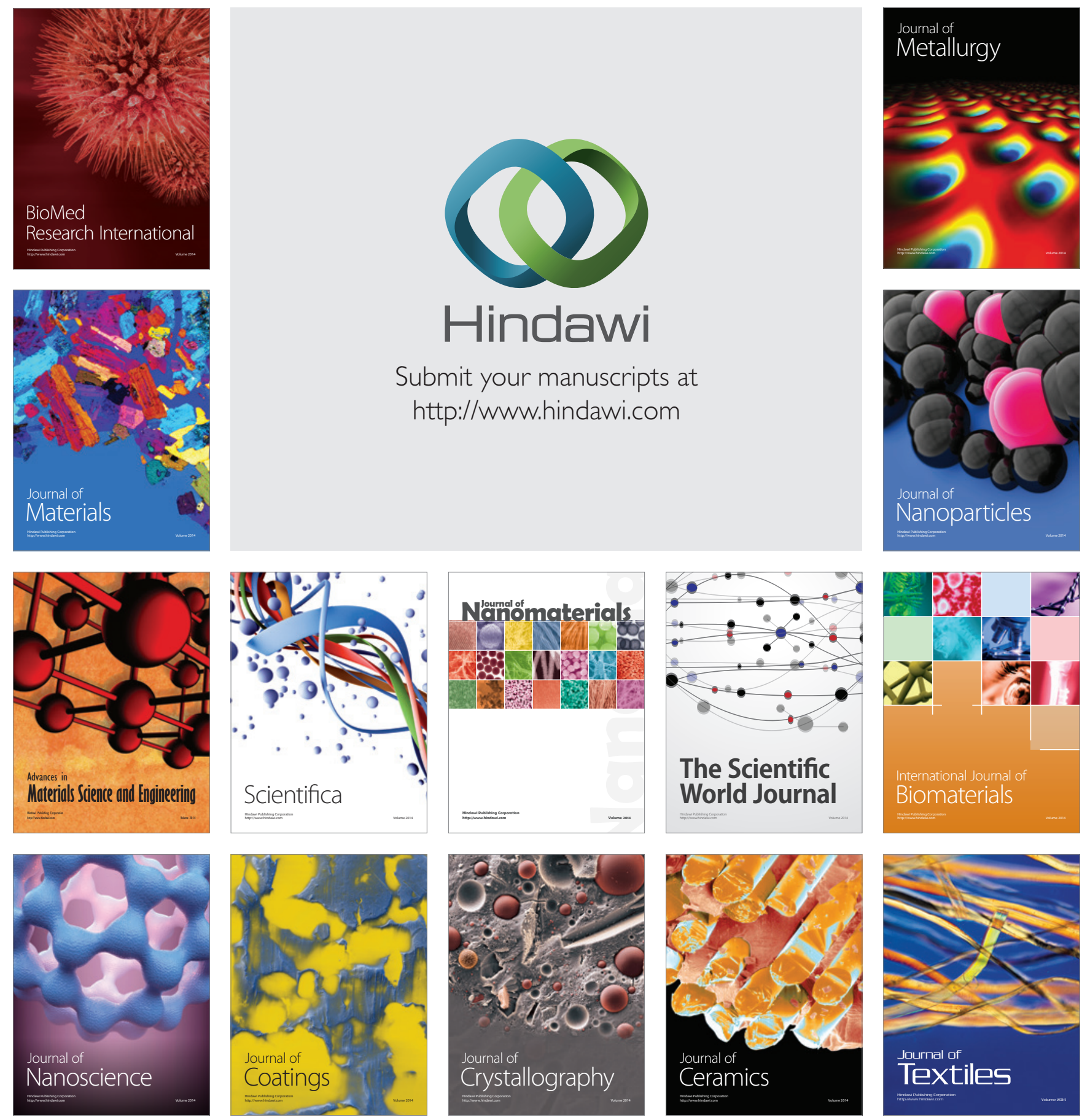